\title{
ON THE COMPUTATION OF CLASS NUMBERS OF REAL ABELIAN FIELDS
}

\author{
TUOMAS HAKKARAINEN
}

\begin{abstract}
In this paper we give a procedure to search for prime divisors of class numbers of real abelian fields and present a table of odd primes $<10000$ not dividing the degree that divide the class numbers of fields of conductor $\leq 2000$. Cohen-Lenstra heuristics allow us to conjecture that no larger prime divisors should exist. Previous computations have been largely limited to prime power conductors.
\end{abstract}

\section{INTRODUCTION}

Class numbers of real abelian fields are at least by present-day knowledge very hard to compute in practice. This is because they are so closely related to the fundamental units, which are difficult to compute or even estimate. Rough estimates that exist in turn lead to poor upper bounds for class numbers. Only for fields of small conductors can one bound class numbers decently with Odlyzko's tables of discriminant bounds; using them F. van der Linden 14 was able to determine (assuming GRH in some cases) the class numbers of all the real abelian fields of conductor $\leq 163$. On the other hand, R. Schoof [20] recently predicted, using a heuristic assumption, that class numbers of real abelian fields of prime conductor are most likely very small compared to known upper bounds.

In his work Schoof also presented and applied an efficient method to compute class number divisors in the case of prime conductors. Koyama and Yoshino [1] presented another approach that allows practical computation. The methods also apply to prime power conductors, but for composite conductors (i.e., conductors having different prime divisors) the Galois module structure of (Hasse's) cyclotomic units is more complicated, due to the fact that different subfields may have different conductors, and thus generalizing the method in this direction is more difficult.

Our approach is to study previously known results that allow computations for composite conductors and to combine them with some ideas from the works mentioned above. We present a method to compute class number divisors for any real abelian field and produce a table of such divisors. By heuristic assumptions similar to Schoof's we predict it to contain all odd prime divisors not dividing the degree of the field in question.

H.-W. Leopoldt in his article [13] generalized Kummer's classical results on the divisibility of class numbers to any real abelian field. His main result is that if an

Received by the editor April 28, 2006.

2000 Mathematics Subject Classification. Primary 11R29, 11Y40; Secondary 11R20, 11R27.

Key words and phrases. Class numbers, computation, abelian fields, units.

This work was financially supported by the Turku Centre for Computer Science, TUCS.

(C) 2008 American Mathematical Society Reverts to public domain 28 years from publication 
odd prime $p$ not dividing the degree of the field is a divisor of the class number, then a certain rational product of generalized Bernoulli numbers is divisible by $p$. By applying the $p$-adic class number formula, W. Schwarz 21] was able to give a simple computational criterion equivalent to Leopoldt's criterion, and he computed for all real abelian fields of conductor $f \leq 500$ a table of all primes $p<100000$ which possibly divide the class number. The table shows that for a fixed conductor there are usually roughly 5 to 20 primes satisfying Leopoldt's condition. But Leopoldt actually proved a somewhat deeper fact to be able to state his result, and this is what we apply to sharpen the results of Schwarz. Our procedure also makes it possible to sieve out the actual class number divisors from Schwarz's table.

We will first discuss the group theoretic background of Leopoldt's method by applying some earlier results of Leopoldt [12. This will shed more light on the method of Schwarz. Then we present an additional technique to check if the primes found with Schwarz's method actually come from class numbers. We limit the computation to prime divisors not dividing $2[K: \mathbf{Q}]$, since the primes dividing the degree of the field do not behave similarly and since for the prime 2 there are better techniques available. We mention here however that Schwarz's method could also be used for some primes dividing the degree; indeed, in many cases one could at least prove that a prime dividing the degree does not divide the class number.

For a broader exposition of the present work, see the author's thesis $[8]$.

\section{DeCOMposition OF CLASS NUMBER}

Leopoldt in his thesis 12 presented an arithmetic characterization of a real abelian field, continuing work of Hasse. A main idea was to apply the Wedderburn decomposition of the rational (and later $p$-adic) Galois group ring to the group of units of an abelian field. Leopoldt was able to reduce the study of the class groups of abelian fields with noncyclic Galois group essentially to the cyclic subfields corresponding to the classes of conjugate characters of the field. We review here only the definitions and results necessary for our study.

Let $K$ be a real abelian field of conductor $f$ with Galois group $G$ of order $g$. For $\chi \in \widehat{G}$, denote by $\widehat{\chi}$ a rational-irreducible character of $K$, i.e., $\widehat{\chi}=\sum_{k} \chi^{k}$, where the sum is over the $\mathbf{Q}$-conjugacy class $\tilde{\chi}=\left\{\chi^{k} \mid(k\right.$, ord $\left.\chi)=1\right\}$ of a character $\chi$ of $K$. The values of $\hat{\chi}$ are in Z. Denote by $f_{\chi}, g_{\chi}$ and $\operatorname{Ker} \chi$, respectively, the common conductor, order and kernel of the $\mathbf{Q}$-conjugates of $\chi$. There is a one-toone correspondence between the $\mathbf{Q}$-conjugacy classes of the character group and the cyclic subfields of $K$, given by $\tilde{\chi} \longleftrightarrow\langle\chi\rangle$; denote the cyclic field corresponding to $\tilde{\chi}$ by $K_{\chi}$. Its degree is $g_{\chi}$, its conductor $f_{\chi}$, and $\operatorname{Gal}\left(K_{\chi} / \mathbf{Q}\right)=G_{\chi} \simeq G / \operatorname{Ker} \chi \simeq\langle\chi\rangle$.

The group algebra $\mathbf{Q}[G]=\left\{\sum_{\sigma \in G} a_{\sigma} \sigma \mid a_{\sigma} \in \mathbf{Q}\right\}$ admits the Wedderburn decomposition

$$
\mathbf{Q}[G]=\bigoplus_{\widetilde{\chi}} \mathbf{Q}[G] e_{\tilde{\chi}} \simeq \bigoplus_{\widetilde{\chi}} \mathbf{Q}\left(\zeta_{g_{\chi}}\right)
$$

via the rational orthogonal idempotents $e_{\tilde{\chi}}=\frac{1}{g} \sum_{\sigma \in G} \widehat{\chi}\left(\sigma^{-1}\right) \sigma$. Here and hereafter we use the notation $\zeta_{m}=e^{2 \pi i / m}$ for any $m \in \mathbf{N}$. The maximal order of $\mathbf{Q}[G]$ is $\bigoplus_{\tilde{\chi}} \mathbf{Z}[G] e_{\tilde{\chi}} \simeq \bigoplus_{\tilde{\chi}} \mathbf{Z}\left[\zeta_{g_{\chi}}\right]$ and $\mathbf{Z}[G]$ is of finite index $g \cdot Q_{G}$ in it as a subgroup, with $Q_{G} \in \mathbf{Z}$ containing only primes dividing $g$.

On tensoring by $\mathbf{Q}$, the unit group $E_{K}$ of $K$ may be regarded as a $\mathbf{Q}[G]$-module; thus it decomposes similarly in the form $E_{K}=\bigoplus_{\tilde{\chi}} E_{K}^{e_{\tilde{\chi}}}$. Rather than studying this 
decomposition directly, one introduces a subgroup $E^{K_{+}}$of $E_{K}$ of finite index; see below. Let $N_{K_{\chi} / k}$ denote the norm from $K_{\chi}$ to any subfield $k$, and define in $E_{K_{\chi}}$ the group of $\chi$-relative units

$$
E_{\chi}=\left\{\varepsilon \in E_{K_{\chi}} \mid N_{K_{\chi} / k}(\varepsilon)= \pm 1 \quad \forall k \subsetneq K_{\chi}\right\}
$$

(cf. [8, p. 14]; Leopoldt uses the notation $E_{\tilde{\chi}}^{+}$and the term narrow $\widetilde{\chi}$-relative units). This is a subgroup of the units of $K_{\chi}$ of rank $\varphi\left(g_{\chi}\right)$ (where $\varphi$ is the Euler function), and it has a subgroup of $\chi$-relative cyclotomic units

$$
F_{\chi}=\left\langle-1, \eta^{\tau} \mid \tau \in G_{\chi}\right\rangle
$$

with finite index

$$
h_{\chi}=\left[E_{\chi}: F_{\chi}\right] .
$$

Here the element $\eta$ is defined as follows: Let $H$ be the subgroup of $\left(\mathbf{Z} / f_{\chi} \mathbf{Z}\right)^{\times}$ corresponding to $\operatorname{Gal}\left(\mathbf{Q}\left(\zeta_{f_{\chi}}\right) / K_{\chi}\right)$, and let $H^{+} \subset \mathbf{Z}$ be a system of representatives of $H /\{ \pm 1\}$. Define

$$
\Theta_{\chi}=\prod_{a \in H^{+}}\left(\zeta_{2 f_{\chi}}^{a}-\zeta_{2 f_{\chi}}^{-a}\right), \quad \Lambda_{\chi}=\prod_{\ell \mid g_{\chi}}\left(1-\sigma^{g_{\chi} / \ell}\right)
$$

where $\ell$ runs through all the prime divisors of $g_{\chi}$ and $\sigma$ is a fixed generator of $G_{\chi}$; then $\Theta_{\chi}^{\sigma-1}$ is a unit of $K_{\chi}$ and $\eta=\Theta_{\chi}^{\Lambda_{\chi}}$.

Both $E_{\chi}$ and $F_{\chi}$ depend only on $\widetilde{\chi}$ and thus are independent of the choice of $K$ containing $K_{\chi}$. The groups of absolute values, $\left|E_{\chi}\right| \simeq E_{\chi} /\{ \pm 1\}$ and $\left|F_{\chi}\right| \simeq$ $F_{\chi} /\{ \pm 1\}$, are modules over $\mathbf{Z}\left[G_{\chi}\right] e_{\tilde{\chi}} \simeq \mathbf{Z}\left[\zeta_{g_{\chi}}\right]$. Another characterization of the $\chi$ relative units is that they are the units $\varepsilon \in E_{K_{\chi}}$ satisfying $|\varepsilon|^{e_{\tilde{\chi}}}=|\varepsilon|$. In particular, $|\varepsilon| \in E_{K}^{e \tilde{x}}$.

When considering $E_{\chi}$ as a subgroup of the units of $K$, we see that the direct $\operatorname{sum} E^{K_{+}}=\bigoplus_{\tilde{\chi}}\left|E_{\chi}\right|$ over all the rational characters of $K$ forms a group of units of finite index, say $Q_{K}^{+}$, in the group $E_{K}$. Using this decomposition of the unit group and a similar decomposition of the regulator of $K$, we may split the class number of $K$ in the form (see [12, p. 41])

$$
h_{K}=\frac{Q_{K}^{+}}{Q_{G}} \prod_{\tilde{\chi}} h_{\chi}
$$

where $Q_{K}^{+}$and $Q_{G}$ are rational integers as explained above, and the product runs through the $\mathbf{Q}$-conjugacy classes $\tilde{\chi}$ of $K$. It is in general difficult to compute the number $Q_{K}^{+}$in practice. The quotient $Q_{K}^{+} / Q_{G}$ is usually not integral. The numbers $Q_{K}^{+}$and $Q_{G}$ are comprised only of primes dividing $2 g$, and since we assumed that $p$ is not a divisor of $2 g$, we may conclude that the $p$-part of the class number $h_{K}$ of $K$ is equal to the product of the $p$-parts of $h_{\chi}$ :

$$
h_{K, p}=\prod_{\tilde{\chi}} h_{\chi, p}
$$

Remark 2.1. Leopoldt also shows that the numbers $h_{\chi}$ are norms of some ideals in $\mathbf{Z}\left[\zeta_{g_{\chi}}\right]$. It follows that once $p$ divides $h_{\chi}$, then also $p^{f_{p}}$ divides $h_{\chi}$, where $f_{p}$ is the residue class degree of $p$.

For $p \nmid 2 g$, we have $g^{-1} \equiv a_{k}\left(\bmod p^{k}\right)$ for some $a_{k} \in \mathbf{Z}$ with $k=1,2, \ldots$ By defining $\alpha^{1 / g}=\alpha^{a_{k}}$ for $\alpha \in \mathrm{Cl}_{p}$ of order $p^{k}$, we may split the $p$-primary part $\mathrm{Cl}_{p}$ of 
the class group $\mathrm{Cl}_{K}$ as a module over $\mathbf{Z}[G]$ through the idempotents $e_{\tilde{\chi}}$. We obtain the decomposition (see [12, p. 44])

$$
\mathrm{Cl}_{p}=\bigoplus_{\tilde{\chi}} \mathrm{Cl}_{\chi, p}
$$

where $\mathrm{Cl}_{\chi, p}=\mathrm{Cl}_{p}^{e \tilde{\chi}}$ and $\# \mathrm{Cl}_{\chi, p}=h_{\chi, p}$, the $p$-part of $h_{\chi}$. The $\mathbf{Z}\left[\zeta_{g_{\chi}}\right]$-module $\mathrm{Cl}_{\chi, p}$ depends only on $K_{\chi}$ and can also be characterized as the group of ideal classes of order a power of $p$ in $K_{\chi}$ that satisfy the following condition: any ideal in the ideal class becomes principal under the relative norm map to any subfield $L \varsubsetneqq K_{\chi}$ (see [13]). Thus the values $h_{\chi}$ also provide structural information on the class group.

Leopoldt [13] showed the following fact when proving his theorem about the class number divisibility referred to in the introduction. The proof is based on the decomposition of the $p$-class group, the reflection theorem and the Stickelberger theorem.

Lemma 2.2. Let $p$ be an odd prime dividing neither the conductor nor the degree of the real abelian field $K$ and let $\chi$ be a character of $K$. If $\mathrm{Cl}_{\chi, p} \neq 1$, then

$$
\prod_{\psi \in \widetilde{\chi}} B_{p-1, \psi} \equiv 0 \quad(\bmod p)
$$

where $B_{k, \psi}$ is the kth generalized Bernoulli number associated to $\psi$.

Note that the above product over the $\mathbf{Q}$-conjugacy class $\tilde{\chi}$ of $\chi$ is rational.

Leopoldt also obtained a result in the ramified case $p \mid f, p^{2} \nmid f$, but we omit it from this study for the sake of simplicity; in the computations we dealt with the case $p \mid f$ using another method.

Remark 2.3. There exist more recent results on the decomposition of the class group through rational $p$-adic characters that could allow more precise computations; see for example an article of Aoki [1] on the structure of $p$-adic parts of the class group. But computations with $p$-adic numbers may be more difficult or even impossible to perform in practice (cf. [10]). In order to preserve efficiency of our algorithms, we prefer the rational approach. Schoof, on the other hand, bases his method on Gras's conjecture about the relationship between the $p$-adic parts of the class groups and of the units modulo cyclotomic units, while all his computations are in rational numbers. Gras's conjecture was proved by R. Greenberg in the case $p$ not dividing the degree; he in fact showed that the orders of the $p$-adic parts of the class group and units modulo cyclotomic units coincide. This gives a connection between Schoof's method and ours.

\section{The ALGORITHM}

We first give an outline of the method. As presented in the preceding section, we will omit the prime 2 and the primes dividing the degree $g$ of the field $K$ in question. To check if a prime $p \nmid 2 g$ divides the class number of $K$, it suffices to run the test for all the $h_{\chi, p}$ separately, i.e., it is sufficient to study only cyclic fields $K_{\chi}$ and cyclic modules $\left|F_{\chi}\right|$ of cyclotomic units. When computing $h_{\chi}$, we always choose $K=K_{\chi}$ and $g=g_{\chi}$.

The method consists of three parts. First we put an upper bound for the primes to be tested. For each prime below this bound, we apply Lemma 2.2 and the method of Schwarz [21], and we are left with a small number of primes that must be tested 
further; for all the other primes $p$, the $\chi$-class number is not divisible by $p$. In view of Leopoldt's result, Schwarz's method not only gives the primes $p$ possibly dividing the class number $h_{K}$, but also specifies the $h_{\chi}$ that may admit the divisor $p$.

The second step consists of a search for cyclotomic units that are $p$ th powers in the unit group, extending an idea of van der Linden [14. In this way we are able to eliminate most of the remaining primes; they do not divide $h_{\chi}$.

Passing these tests is a necessary condition for the $p$-divisibility, and after them we have a strong belief that $p$ could divide the $\chi$-class number, but this is still not a proof. To verify the divisibility, we finally check whether the $p$ th root of a unit found in the second step is in $K_{\chi}$. We use a method presented in an article of G. Gras and M.-N. Gras [5].

Moreover, we provide a method to check whether $h_{\chi}$ is divisible by a higher power of $p$. This is also based on [5].

We limited the search to the fields of conductor $f \leq 2000$ and to the primes $p<10000$. In theory there could be larger primes dividing these class numbers, but we will see that the heuristics of Cohen and Lenstra 3 and the results of the computations (the largest prime factor found was 379 ) show this to be very unlikely.

\section{SCHWARZ'S METHOD}

We now describe the first step of the computation. Let $K_{0}=\mathbf{Q}\left(\zeta_{f}+\zeta_{f}^{-1}\right)$ be the maximal real abelian field of conductor $f$. As is clear from the preceding discussion, to study the $p$-divisibility of the class numbers of real abelian fields of conductor $f$, we have to compute the $(\chi, p)$-parts $h_{\chi, p}$ of the class number of $K_{0}$ for all the Q-conjugacy classes of characters $\chi$ of $K_{0}$.

Let $\chi$ be a character of $K_{0}$. Since $h_{\chi}$ is independent of the choice of the field containing $K_{\chi}$, we may always assume $f$ to be chosen minimal, i.e., $f=f_{\chi}$. In the first step we also assume $p \nmid f$; the primes dividing $f$ will be checked in the second step of the algorithm. We choose a bound for the primes $p \nmid 2 f g_{\chi}$ to test.

Denote by $[a]$ the integer part of $a>0$. We begin with a lemma [21, pp. 45-46].

Lemma 4.1. If $\chi$ is a character of conductor $f$ and order $n$ and $p \nmid 2 f$ is a prime, then

$$
B_{p-1, \chi} \equiv-\chi(p) \sum_{i=1}^{f-1} \chi(i) \sum_{\nu=1}^{\left[\frac{p i}{f}\right]} \nu^{-1} f^{-1} \quad\left(\bmod \mathcal{P}_{\chi}\right)
$$

for a prime ideal $\mathcal{P}_{\chi} \mid p$ in $\mathbf{Z}\left[\zeta_{n}\right]$.

Proof. We sketch a proof. Fix an embedding of the field of all algebraic numbers in an algebraic closure $\boldsymbol{\Omega}_{p}$ of the $p$-adic field $\mathbf{Q}_{p}$ and regard all algebraic elements as being in $\boldsymbol{\Omega}_{p}$. The congruence $\alpha \equiv \beta\left(\bmod p^{n}\right)$ with $\alpha, \beta \in \boldsymbol{\Omega}_{p}$ means that the $p$-exponent of $\alpha-\beta$ is $\geq n$. Write shortly $\zeta_{f}=\zeta$.

By using properties of $p$-adic $L$-functions $L_{p}(s, \chi)$ we have

$$
B_{p-1, \chi} \equiv L_{p}(2-p, \chi) \equiv L_{p}(1, \chi) \quad(\bmod p) .
$$

Metsänkylä [16] shows that

$$
L_{p}(1, \chi) \equiv-\sum_{i=1}^{f-1} b_{i} \chi(i) \quad(\bmod p)
$$


whenever $b_{i}$ modulo $p$ are rational integers satisfying

$$
\lambda(\zeta)=\frac{(\zeta-1)^{p}-\left(\zeta^{p}-1\right)}{p\left(\zeta^{p}-1\right)} \equiv \sum_{i=1}^{f-1} b_{i} \zeta^{i} \quad(\bmod p) .
$$

(By Schwarz, p. 43, the number $\lambda(\zeta)$ modulo $p$ equals the Fermat quotient of $\zeta^{p}-1$. )

Let $a \in \mathbf{Z}, a \equiv p^{-1}(\bmod f)$. Since $\frac{1}{p}\left(\begin{array}{l}p \\ k\end{array}\right) \equiv \frac{1}{k}(\bmod p)$, we may write

$$
(1-\zeta) \lambda\left(\zeta^{a}\right) \equiv \sum_{\mu=0}^{f-1} c_{\mu} \zeta^{\mu} \quad(\bmod p)
$$

with

$$
c_{\mu} \equiv \sum_{\substack{k=1 \\ a k \equiv \mu(\bmod f)}}^{p-1} k^{-1} \equiv \sum_{\nu=\left[\frac{p(\mu-1)}{f}\right]+1}^{\left[\frac{p \mu}{f}\right]} \nu^{-1} f^{-1}(\bmod p) .
$$

Define the numbers $b_{i}$ for all $i \in \mathbf{Z} \backslash f \mathbf{Z}$ by periodicity modulo $f$. We have

$$
(1-\zeta) \lambda\left(\zeta^{a}\right) \equiv(1-\zeta) \sum_{i=1}^{f-1} b_{p i} \zeta^{i} \equiv \sum_{i=1}^{f-1}\left(b_{p i}-b_{p(i-1)}\right) \zeta^{i} \quad(\bmod p)
$$

Consequently, by choosing

$$
b_{p i} \equiv \sum_{\nu=1}^{\left[\frac{p i}{f}\right]} \nu^{-1} f^{-1} \quad(\bmod p),
$$

the $b_{i}$ satisfy the requirement. By the formula (4.2),

$$
L_{p}(1, \chi) \equiv-\sum_{i=1}^{f-1} b_{p i} \chi(p i) \quad(\bmod p) .
$$

We conclude that the congruence (4.1) holds modulo $p$ (in $\boldsymbol{\Omega}_{p}$ ). The claim follows since the numbers in (4.1) are $p$-integers in the field $\mathbf{Q}\left(\zeta_{n}\right)$.

Denote by $\Phi_{n}(x)$ the $n$th cyclotomic polynomial.

Proposition 4.1. Let $f$ be the conductor and $n$ the order of $\chi$. Let

$$
\lambda:(\mathbf{Z} / f \mathbf{Z})^{\times} \rightarrow\{0, \ldots, n-1\}
$$

be defined by $\chi(i)=\zeta_{n}^{\lambda(i)}$. If the prime $p \nmid 2 f n$ divides the $\chi$-class number $h_{\chi}$, then

$$
\operatorname{GCD}_{\mathbf{F}_{p}[x]}\left(\sum_{\substack{i=1 \\(i, f)=1}}^{f-1} a_{i} x^{\lambda(i)}, \Phi_{n}(x)\right) \neq \overline{1}
$$

where $a_{i} \equiv \sum_{\nu=1}^{\left[\frac{p i}{f}\right]} \nu^{-1} f^{-1}(\bmod p)$ and $\operatorname{GCD}_{\mathbf{F}_{p}[x]}$ denotes the greatest common divisor of the indicated polynomials, regarded as polynomials over $\mathbf{F}_{p}$.

Proof. Assume $p \mid h_{\chi}$. By Lemma 2.2. $\prod_{\chi \in \widetilde{\chi}} B_{p-1, \chi} \equiv 0(\bmod p)$. Hence it follows from (4.1) that

$$
\prod_{\chi \in \tilde{\chi}} \sum_{i=1}^{f-1} a_{i} \chi(i) \equiv 0 \quad(\bmod p)
$$


Since the conjugates $\chi^{\sigma}$ of $\chi$ satisfy $\chi^{\sigma}(i)=\zeta_{n}^{k \lambda(i)}$ and the zeros of $\Phi_{n}(x)$ are $\zeta_{n}^{k}$ for $(k, n)=1$, we have

$$
\prod_{\chi \in \tilde{\chi}} \sum_{\substack{i=1 \\(i, f)=1}}^{f-1} a_{i} \chi(i)=\prod_{\substack{k=1 \\(k, n)=1}}^{n-1} \sum_{\substack{i=1 \\(i, f)=1}}^{f-1} a_{i} \zeta_{n}^{k \lambda(i)}=\operatorname{Res}\left(\Phi_{n}(x), \sum_{\substack{i=1 \\(i, f)=1}}^{f-1} a_{i} x^{\lambda(i)}\right),
$$

where $\operatorname{Res}(\cdot, \cdot)$ denotes the resultant. Finally, $p$ divides $\operatorname{Res}(f(x), g(x))$ if and only if $\operatorname{GCD}_{\mathbf{F}_{p}[x]}(f(x), g(x)) \neq \overline{1}$. The claim follows.

The proof of the proposition is essentially found in Schwarz's thesis. Schwarz also shows that the computational complexity of the method is $O\left(p+f+n^{2}\right)$. He used the result to produce a table of possible class number divisors $p<100000$ for any real abelian field of conductor $f \leq 500$. By resorting to Leopoldt's decomposition of class number, the results become more transparent in the case of composite conductor. In particular, we know explicitly the factor group of units that is of order $h_{\chi}$.

Remark 4.2. The $p$-adic class number formula implies that the primes $p \nmid f g_{\chi}$ satisfying (4.3) but not dividing the class number must satisfy $v_{p}\left(R_{p}\left(K_{\chi}\right)\right) \geq g_{\chi}$, where $v_{p}$ denotes the normalized $p$-adic valuation and $R_{p}\left(K_{\chi}\right)$ is the $p$-adic regulator of the field $K_{\chi}$ (trivially $\left.v_{p}\left(R_{p}\left(K_{\chi}\right)\right) \geq g_{\chi}-1\right)$. In this way we obtain some knowledge of the $p$-adic regulator without knowing the fundamental units. In many cases one could also use the method and the $p$-adic class number formula to check whether the class number is not divisible by a prime dividing the degree of the field. With slight changes to the preceding method, one could also compute the $p$-exponent of the (p-adic) product $h_{K} R_{p}(K)$, thus obtaining an upper bound for the $p$-exponent of the class number.

\section{SECOND STEP}

In [14] van der Linden introduced a method with which he could show by computation that $p \nmid h_{K}$ in some cases. However, his use of the group of units modulo (Hasse's) cyclotomic units is problematic in general, since one may need to combine unit groups of subfields in order to obtain groups of full rank (see [22, p. 150]). We avoid this problem by applying a similar procedure to the groups $E_{\chi} / F_{\chi}$.

To check if $h_{\chi, p} \neq 1$, we need to analyze the structure of the group $E_{\chi} / F_{\chi}$. As noted before, $E_{\chi} /\{ \pm 1\}$ and $F_{\chi} /\{ \pm 1\}$ are $\mathbf{Z}\left[\zeta_{g_{\chi}}\right]$-modules. Recalling that $( \pm \varepsilon)^{e} \tilde{\chi}=$ $\pm \varepsilon$ for any $\varepsilon \in E_{\chi}$ and $\mathbf{Z}\left[G_{\chi}\right] e_{\tilde{\chi}} \simeq \mathbf{Z}\left[\zeta_{g_{\chi}}\right]$, we may also regard $\left|E_{\chi}\right|$ and $\left|F_{\chi}\right|$ as $\mathbf{Z}\left[G_{\chi}\right]$-modules. Thus $F_{\chi} / F_{\chi}^{p}$ admits an $\mathbf{F}_{p}\left[G_{\chi}\right]$-module structure.

The map $x F_{\chi} \mapsto x^{p} F_{\chi}^{p}$ defines an isomorphism $\left(E_{\chi} / F_{\chi}\right)_{p} \simeq\left(E_{\chi}^{p} \cap F_{\chi}\right) / F_{\chi}^{p}$, where $\left(E_{\chi} / F_{\chi}\right)_{p}$ is the $p$-elementary subgroup (the group of elements of order 1 or $p)$. The group $\left(E_{\chi}^{p} \cap F_{\chi}\right) / F_{\chi}^{p}$ is an $\mathbf{F}_{p}\left[G_{\chi}\right]$-submodule of $F_{\chi} / F_{\chi}^{p}$. If nontrivial, it must contain a minimal submodule of $F_{\chi} / F_{\chi}^{p}$. Let this be $F_{i} / F_{\chi}^{p}$; then we have $F_{i} \subseteq E_{\chi}^{p}$. On the other hand, if $F_{j} / F_{\chi}^{p}$ is any minimal submodule of $F_{\chi} / F_{\chi}^{p}$ such that $F_{j} \subseteq E_{\chi}^{p}$, then $F_{j} / F_{\chi}^{p}$ is a submodule of $\left(E_{\chi}^{p} \cap F_{\chi}\right) / F_{\chi}^{p}$. Since the intersection of two different minimal submodules is zero, the $p$-exponent of $h_{\chi}$ is at least the number of minimal submodules $F_{i} / F_{\chi}^{p}$ satisfying $F_{i} \subseteq E_{\chi}^{p}$.

In order to prove that $h_{\chi, p}=1$, it suffices to compute all the minimal submodules of $F_{\chi} / F_{\chi}^{p}$ and to check that all of them contain elements that are not $p$ th powers of units. This is not difficult since the minimal submodules are cyclic and easily 
determined by the following proposition and remark. Recall that the $\mathbf{Z}\left[G_{\chi}\right]$-module $\left|F_{\chi}\right|$ is generated by $\pm \eta=\left( \pm \Theta_{\chi}\right)^{\Lambda_{\chi}}$, where $\Theta_{\chi}$ and $\Lambda_{\chi}$ are defined by (2.1).

Proposition 5.1. Assume that $p \equiv 1\left(\bmod g_{\chi}\right)$. The minimal $\mathbf{F}_{p}\left[G_{\chi}\right]$-submodules of $F_{\chi} / F_{\chi}^{p}$ are $\left\langle\eta^{\Phi_{g_{\chi}}(\sigma) /(\sigma-i)}\right\rangle$, where $i$ runs through all the zeros of $\Phi_{g_{\chi}}(x)(\bmod p)$ and $\sigma$ is a generator of $G_{\chi}$.

Proof. Consider the $\mathbf{F}_{p}\left[G_{\chi}\right]$-homomorphism

$$
\tau: \mathbf{F}_{p}\left[G_{\chi}\right] \rightarrow F_{\chi} / F_{\chi}^{p}, \quad \delta \mapsto \eta^{\delta} F_{\chi}^{p} .
$$

It is obviously well-defined and surjective. Its kernel is an $\mathbf{F}_{p}\left[G_{\chi}\right]$-module, i.e., an ideal in the principal ideal ring $\mathbf{F}_{p}\left[G_{\chi}\right] \simeq \mathbf{F}_{p}[x] /\left\langle x^{g_{\chi}}-1\right\rangle$. Since $F_{\chi}$ is of finite index in $E_{\chi}$, the Z-rank of $\left|F_{\chi}\right|$ is equal to $\varphi\left(g_{\chi}\right)$, thus the $\mathbf{F}_{p^{-}}$-rank of $F_{\chi} / F_{\chi}^{p}$ is $\varphi\left(g_{\chi}\right)$.

Trivially $\Theta_{\chi}^{\sigma^{g_{\chi}}-1}= \pm 1$. Write $\sigma^{g_{\chi}}-1=\prod_{d \mid g_{\chi}} \Phi_{d}(\sigma)$. It follows that $\Lambda_{\chi}$ is divisible by all the $\Phi_{d}(\sigma)$ with $d \neq g_{\chi}$, whence $\eta^{\Phi_{g_{\chi}}(\sigma)}= \pm 1$. Consequently, the kernel $\operatorname{Ker}(\tau)=\left\langle\Phi_{g_{\chi}}(\sigma)\right\rangle$.

By the assumption on $p$, the cyclotomic polynomial $\Phi_{g_{\chi}}(x)$ factors completely modulo $p$ and we have the evident $\mathbf{F}_{p}\left[G_{\chi}\right]$-isomorphisms

$$
\mathbf{F}_{p}\left[G_{\chi}\right] /\left\langle\Phi_{g_{\chi}}(\sigma)\right\rangle \simeq \mathbf{F}_{p}[x] /\left\langle x^{g_{\chi}}-1, \Phi_{g_{\chi}}(x)\right\rangle \simeq \mathbf{F}_{p}[x] /\left\langle\Phi_{g_{\chi}}(x)\right\rangle \simeq \mathbf{F}_{p}^{\varphi\left(g_{\chi}\right)} .
$$

The minimal submodules of $\mathbf{F}_{p}^{\varphi\left(g_{\chi}\right)}$ are $\langle(1,0, \ldots, 0)\rangle, \ldots,\langle(0, \ldots, 0,1)\rangle$. By the isomorphism, they correspond to the modules $\left\langle\Phi_{g_{\chi}}(\sigma) /(\sigma-i)\right\rangle$ in $\mathbf{F}_{p}\left[G_{\chi}\right] /\left\langle\Phi_{g_{\chi}}(\sigma)\right\rangle$, where $\sigma-i$ runs through the factors of $\Phi_{g_{\chi}}(\sigma)(\bmod p)$. The claim follows.

Remark 5.1. The proposition generalizes to all odd primes not dividing $g_{\chi}$. Indeed, choose the smallest $f_{p} \geq 1$ such that $p^{f_{p}} \equiv 1\left(\bmod g_{\chi}\right)$. The $g_{\chi}$ th cyclotomic polynomial factors over $\mathbf{F}_{p}$ into $\varphi\left(g_{\chi}\right) / f_{p}$ distinct polynomials $f_{i}(x)$ of degree $f_{p}$, hence $\mathbf{F}_{p}\left[G_{\chi}\right] /\left\langle\Phi_{g_{\chi}}(\sigma)\right\rangle \simeq\left(\mathrm{GF}\left(p^{f_{p}}\right)\right)^{\varphi\left(g_{\chi}\right) / f_{p}}$. Then the minimal submodules of $F_{\chi} / F_{\chi}^{p}$ are $\left\langle\eta^{\Phi_{g_{\chi}}(\sigma) / f_{i}(\sigma)}\right\rangle$.

Note that if a prime $p$ of order $f_{p}$ modulo $g_{\chi}$ divides $h_{\chi}$, then $p^{f_{p}}$ also divides $h_{\chi}$. This follows from Remark 2.1

To examine if $F_{i} \subseteq E_{\chi}^{p}$, it thus suffices to check whether $\eta^{\Phi_{g_{\chi}}(\sigma) / f_{i}(\sigma)}$ is the $p$ th power of some $\varepsilon \in E_{\chi}$. We explain how this will be done, following 14]. Later we will also need the fact that $\varepsilon \notin F_{\chi}$; this follows from the nontriviality of $F_{i} / F_{\chi}^{p}$.

Choose a prime $q \equiv 1\left(\bmod 2 p f_{\chi}\right)$ and some $b \in \mathbf{Z}$ satisfying the conditions $b^{2 f_{\chi}} \equiv 1(\bmod q), b \neq \equiv 1(\bmod q)$. Then $\zeta_{2 f_{\chi}} \equiv b(\bmod \mathcal{Q})$ for some prime ideal $\mathcal{Q}$ above $q$ in $\mathbf{Q}\left(\zeta_{2 f_{\chi}}\right)$. By writing $\eta^{\Phi_{g_{\chi}}(\sigma) / f_{i}(\sigma)}$ as a rational function $r\left(\zeta_{2 f_{\chi}}\right)$, we examine whether

$$
r(b)^{\frac{q-1}{p}} \equiv 1 \quad(\bmod q) .
$$

Indeed, this must hold if $r\left(\zeta_{2 f_{\chi}}\right)=\varepsilon^{p}$. If the congruence holds, we choose another pair $(q, b)$ and repeat the test; if the congruence condition is not satisfied for some pair, we conclude that $F_{i} \nsubseteq E_{\chi}^{p}$. If for every submodule $F_{i}$ there exists a pair $(q, b)$ not satisfying the congruence, we have the result $p \nmid h_{\chi}$. Otherwise, if there is a prime $p$ and a submodule $F_{i}$ which pass the congruence test for many pairs, this gives strong evidence that $p$ would divide the class number. But since this process involves uncertainty, we still have to apply another method. 
Remark 5.2. Instead of $\zeta_{2 f_{\chi}}$, we may actually use $f_{\chi}$ th roots of 1 in the computations of the second step. In fact, it is an easy exercise to see that $\Theta_{\chi}^{\sigma-1}$ may always be written as a rational function of $\zeta_{f_{\chi}}$.

\section{THIRD STEP}

For some $\alpha=\eta^{\Phi_{g_{\chi}}(\sigma) / f_{i}(\sigma)}$ satisfying (5.1) for many pairs $(q, b)$, we want to verify that $\alpha$ is a $p$ th power in $E_{\chi}$. This is equivalent to showing that $\sqrt[p]{\alpha}$ is an element of $K_{\chi}$. As a unit of $K_{\chi}$, the element $\alpha$ has $g_{\chi}$ conjugates in $K_{\chi}$. We calculate an approximation of $\alpha$ and its conjugates $\alpha^{\sigma}$ as real numbers by noting that

$$
\frac{\zeta_{2 f}^{a}-\zeta_{2 f}^{-a}}{\zeta_{2 f}-\zeta_{2 f}^{-1}}=\frac{\sin (a \pi / f)}{\sin (\pi / f)}
$$

If the polynomial $m_{p}(x)=\prod_{\sigma}\left(x-\sqrt[p]{\alpha^{\sigma}}\right)$ has integral coefficients, then $\alpha$ is a $p$ th power; this is the minimum polynomial of $\sqrt[p]{\alpha}$. Then also $\sqrt[p]{\alpha^{\sigma}}=\sqrt[p]{\alpha} \sigma$ and $\sqrt[p]{\alpha} \in K_{\chi}$. But since we have used only approximations, this is still not a proof.

Denote by $\widetilde{m}_{p}$ the polynomial that we have computed in this way to approximate $m_{p}$. If some coefficient of $\widetilde{m}_{p}$ is not close to an integer, this shows that $\alpha$ is not a $p$ th power, given that the precision in the computations is adequate. Otherwise, if all the coefficients of $\widetilde{m}_{p}$ are very close to integers, we round off the coefficients to obtain the supposed minimum polynomial $m_{p}(x) \in \mathbf{Z}[x]$. We then check whether $m_{p}(x) \mid m\left(x^{p}\right)$, where $m(x)$ is the minimum polynomial of $\alpha$. If this holds, it finally proves that $m_{p}$ is the minimum polynomial of $\sqrt[p]{\alpha}$ and that $\sqrt[p]{\alpha}$ is an element of $K_{\chi}$.

Since we actually compute $\alpha$ in $F_{\chi} / F_{\chi}^{p}$, note that we may minimize modulo $p$ the absolute values of the coefficients of $\Phi_{g_{\chi}}(x) / f_{i}(x) \in \mathbf{Z}[x]$ in order to prevent coefficient explosion.

\section{Higher POWERS OF $p$}

Suppose that using the preceding method we have found a prime $p$ with $p \mid h_{\chi}$. We want to check whether $h_{\chi}$ is divisible by a higher power of $p$. G. Gras and M.-N. Gras [5] introduced a method with which this verification is in principle possible.

The following lemma describes the correspondence we found between our and Gras's approach. By combining this result with our method as shown later, we were able to check all the cases with $p \equiv 1\left(\bmod g_{\chi}\right)$ encountered in the computations.

Lemma 7.1. Let $n \geq 2$ and assume $p \equiv 1(\bmod n)$. Let $k \in \mathbf{Z}$ be a zero of $\Phi_{n}(x)$ modulo $p$. We have

$$
\frac{\Phi_{n}\left(\zeta_{n}\right)}{\zeta_{n}-k} \equiv \pm \frac{N\left(\zeta_{n}-k\right)}{\zeta_{n}-k} \quad\left(\bmod p \mathbf{Z}\left[\zeta_{n}\right]\right)
$$

where $N(\gamma)$ denotes the absolute norm of $\gamma \in \mathbf{Z}\left[\zeta_{n}\right]$.

Proof. By the assumption on $p$, all the zeros of $\Phi_{n}(x)(\bmod p)$ are of the form $k^{j}$, where $(j, n)=1$. Thus the prime ideals of $\mathbf{Z}\left[\zeta_{n}\right]$ above $p$ are $\mathcal{P}_{j}=\left\langle p, \zeta_{n}-k^{j}\right\rangle$, $(j, n)=1$. Write the claim in the form

$$
\prod_{\substack{j=2 \\(j, n)=1}}^{n}\left(\zeta_{n}-k^{j}\right) \equiv \pm \prod_{\substack{j=2 \\(j, n)=1}}^{n}\left(\zeta_{n}^{j}-k\right) \quad\left(\bmod p \mathbf{Z}\left[\zeta_{n}\right]\right) .
$$


Since $\zeta_{n} \equiv k\left(\bmod \mathcal{P}_{1}\right)$, this congruence holds modulo $\mathcal{P}_{1}$. Moreover, since the automorphisms $\zeta_{n} \mapsto \zeta_{n}^{j},(j, n)=1$, permute the prime ideals, we see for any $i \neq 1$ that both products contain a factor divisible by $\mathcal{P}_{i}$.

Assume $p \mid h_{\chi}$ and $p \equiv 1\left(\bmod g_{\chi}\right)$ and let $\sigma$ be a fixed generator of $G_{\chi}$. Let $N(\sigma-k)=\prod_{j=1,\left(j, g_{\chi}\right)=1}^{g_{\chi}}\left(\sigma^{j}-k\right) \in \mathbf{Z}\left[G_{\chi}\right]$. By the isomorphism $\mathbf{Z}\left[\zeta_{g_{\chi}}\right] \simeq$ $\mathbf{Z}\left[G_{\chi}\right] /\left\langle\Phi_{g_{\chi}}(\sigma)\right\rangle$ and the lemma, we write in $\mathbf{Z}\left[G_{\chi}\right]$,

$$
\frac{\Phi_{g_{\chi}}(\sigma)}{\sigma-k} \equiv \pm \frac{N(\sigma-k)}{\sigma-k} \quad\left(\bmod p, \Phi_{g_{\chi}}(\sigma)\right) .
$$

Hence the isomorphism induced by $\tau$ in the proof of Proposition 5.1 implies that $\eta^{\Phi_{g_{\chi}}(\sigma) /(\sigma-k)}$ is a $p$ th power in $E_{\chi}$ only if $\eta^{N(\sigma-k) /(\sigma-k)}$ is a $p$ th power in $E_{\chi}$. We know that $N(\sigma-k) \equiv p m\left(\bmod \Phi_{g_{\chi}}(\sigma)\right)$ with $p \nmid m$ (if $p \mid m$, change $k$ to some $k+t p$ until $p \nmid m$; this was possible in all the cases we confronted in the computations). Thus we have $\eta^{p m /(\sigma-k)}=\varepsilon^{p}$ for some $\varepsilon \in E_{\chi} \backslash F_{\chi}$ (see the paragraph after Remark 5.1). From this it follows that $\varepsilon^{\sigma-k}=\eta^{m}$.

Let $F_{\chi}^{\prime}=\left\langle-1, \varepsilon^{\tau} \mid \tau \in G_{\chi}\right\rangle$. Then $\left|F_{\chi}^{\prime}\right|$ is a $\mathbf{Z}\left[G_{\chi}\right]$-module. Since $\varepsilon \notin F_{\chi}$, but $\varepsilon^{p} \in F_{\chi}$ and $\varepsilon^{\sigma}=\varepsilon^{k} \eta^{m}$, we have $\left[F_{\chi} F_{\chi}^{\prime}: F_{\chi}\right]=p$. On the other hand, $p \nmid\left[F_{\chi} F_{\chi}^{\prime}: F_{\chi}^{\prime}\right]$ since $\eta^{m} \in F_{\chi}^{\prime}$ and $F_{\chi}^{\prime}$ is closed under $\sigma$-conjugation. Knowing that $p \mid\left[F_{\chi} F_{\chi}^{\prime}: F_{\chi}^{m}\right]$, we thus deduce $\left[F_{\chi}^{\prime}: F_{\chi}^{m}\right]=p u$ with some $u \in \mathbf{Z}, p \nmid u$. Finally, since $\left[E_{\chi}: F_{\chi}^{m}\right]=\left[E_{\chi}: F_{\chi}\right]\left[F_{\chi}: F_{\chi}^{m}\right]<\infty$, we conclude that $\left[E_{\chi}: F_{\chi}^{\prime}\right]<\infty$ and that the $p$-exponent of $\left[E_{\chi}: F_{\chi}^{\prime}\right]$ is equal to the $p$-exponent of $h_{\chi} / p$.

Now we run the third step using $F_{\chi}^{\prime}$ in place of $F_{\chi}$. Proposition 5.1 holds with $\varepsilon$ in place of $\eta$. We thus check whether $\varepsilon^{\Phi_{g_{\chi}}(\sigma) /(\sigma-j)}$ is a $p$ th power for any $j$ satisfying $\Phi_{g_{\chi}}(j) \equiv 0(\bmod p)$. By (7.1), this is equivalent to checking whether $\varepsilon^{N(\sigma-j) /(\sigma-j)}$ is a $p$ th power. We may compute $\varepsilon=\sqrt[p]{\eta^{N(\sigma-j) /(\sigma-j)}}$ and its conjugates $\varepsilon^{\sigma^{k}}$ with a sufficient precision. It follows that we may compute an approximation of any conjugate of $\varepsilon^{\Phi_{g_{\chi}}(\sigma) /(\sigma-j)}$.

In fact, one knows a priori that it suffices to check only those minimal submodules of $F_{\chi}^{\prime} / F_{\chi}^{\prime} p$ that correspond to the minimal submodules of $F_{\chi} / F_{\chi}^{p}$ found to contain $p$ th powers. Indeed, assume

$$
\varepsilon \in E_{\chi} \backslash F_{\chi}, \quad \varepsilon^{p}=\eta^{N(\sigma-i) /(\sigma-i)} ; \quad \rho \in E_{\chi} \backslash F_{\chi}^{\prime}, \quad \rho^{p}=\varepsilon^{N(\sigma-j) /(\sigma-j)},
$$

where $i \neq j$. Let $\varepsilon_{1}$ be the real number defined by $\varepsilon_{1}^{p}=\eta^{N(\sigma-j) /(\sigma-j)}$. If $N(\sigma-i)=$ $p m_{1}$ with $p \nmid m_{1}$, we have $\eta^{m_{1}}=\varepsilon^{\sigma-i}$, so $\varepsilon_{1}^{m_{1}}=\rho^{\sigma-i} \in E_{\chi}$. Since trivially $\varepsilon_{1}^{p} \in E_{\chi}$ and $\left(p, m_{1}\right)=1$, we conclude $\varepsilon_{1} \in E_{\chi}$.

This method seems to fail for $p \not \equiv 1\left(\bmod g_{\chi}\right)$. Indeed, the second step only gives us $p$ th powers explicitly, although we know by the theory that there also exist $p^{f_{p}}$ th powers, where $f_{p}$ is the residue class degree. Nevertheless, if we find in the second step that $p \mid h_{\chi}$, we may check whether the number $\varepsilon \in \mathbf{R}$ satisfying $\varepsilon^{p^{f_{p}}}=\eta^{N\left(f_{i}(\sigma)\right) / f_{i}(\sigma)}$ belongs to $E_{\chi} \backslash F_{\chi}$ for some $i$. In this way we may still find a $p^{f_{p}}$ th power in $E_{\chi}$, but whether this happens remains theoretically unproven since there is no result similar to (7.1). In the computations this was possible in all the cases we confronted; indeed, the results in [5] give evidence that this should always be the case. Choose again $\left\langle-1, \varepsilon^{\tau} \mid \tau \in G_{\chi}\right\rangle=F_{\chi}^{\prime}$. A similar reasoning as above shows that the $p$-exponent of $\left[E_{\chi}: F_{\chi}^{\prime}\right]$ is equal to the $p$-exponent of $h_{\chi} / p^{f_{p}}$. 
Finally, using the second and third steps (with $F_{\chi}^{\prime}$ in place of $F_{\chi}$ ), we can check whether $p \mid\left(h_{\chi} / p^{f_{p}}\right)$.

In this way we were able to verify that among the fields of conductor at most 2000 there are only the following two cases in which $h_{\chi}$ contains $p^{f_{p}}$ more than once (both with $f_{p}=1$ ). The 17-class number of a 16-degree field of conductor 1921 is $17^{3}$ and the 3-class number of the quadratic field of prime conductor 1129 is $3^{2}$. The latter is also found in Schoof's table [20. Additionally, we verified that all the other higher powers of $p$ found in his table could also be determined with our method.

Remark 7.2. G. Gras and M.-N. Gras [5] computed class numbers of real abelian fields of small degree using a method quite similar to our method of finding $p$ th powers. They also used Leopoldt's condition similar to Schwarz's method to limit the number of possible divisors. The tables [6] and [7] were computed using this method. The aim in [5] was to compute class numbers of real abelian fields using explicit upper bounds that are practical only in fields of small degree; hence the efficiency of the algorithm was not as crucial as in our computations. On the other hand, the efficiency might be improved using first the congruence method as in the second step. Gras's method essentially consists of a search of units of $E_{\chi}^{\mathcal{P}}$ belonging to $F_{\chi}$, where $\mathcal{P}$ is a prime ideal of $\mathbf{Z}\left[\zeta_{g_{\chi}}\right]$ above $p$; this amounts to searching for units of the form $\left(\eta^{N\left(f_{i}(\sigma)\right) / f_{i}(\sigma)}\right)^{1 / p^{f} p}$ with $\mathcal{P}=\left\langle p, f_{i}\left(\zeta_{g_{\chi}}\right)\right\rangle$. This suggests that our method could similarly be generalized to search (by the isomorphism $\mathbf{Z}\left[\zeta_{g_{\chi}}\right] \simeq$ $\left.\mathbf{Z}\left[G_{\chi}\right] /\left\langle\Phi_{g_{\chi}}(\sigma)\right\rangle\right)$ for $\mathcal{P}$ th powers in $E_{\chi}$. This would settle more naturally the case of a larger residue class degree. One possibility would be to investigate the group $\left(E_{\chi} / F_{\chi}\right)_{\mathcal{P}}$ (the $\mathcal{P}$-part will be defined later in this work).

\section{An example of the Calculation}

The following example shows how the calculations were done. Choose $f=$ $1261=13 \cdot 97$. Let $K=\mathbf{Q}\left(\zeta_{f}+\zeta_{f}^{-1}\right)$. There are 47 real cyclic fields of conductor $f$ corresponding to the nontrivial $\mathbf{Q}$-conjugacy classes of characters of $K$.

We run for any $h_{\chi}$ the first step of the method by checking whether the condition (4.3) holds. All the necessary information for the computation may be gathered from the knowledge of the corresponding $\mathbf{Q}$-conjugacy class $\tilde{\chi}$. This is the lengthy part of the calculation since we check all the primes $2<p<10000, p \nmid f$, for all the 47 different $h_{\chi}$. We find out that there are in total 68 primes (counted with multiplicity) that satisfy (4.3) for some $h_{\chi}$, of which 10 primes divide $g_{\chi}$. We continue to the second step only with the primes not dividing $g_{\chi}$ (the 10 discarded primes of course would also contain some information of the class number divisibility, but they would require another method). Usually the number of primes satisfying (4.3) was found to be roughly proportional to the number of different $h_{\chi}$.

In the second step we check all the remaining 58 cases. We also check for all different $h_{\chi}$ the primes 13 and 97 dividing $f$. There are a total of 152 pairs $\left(h_{\chi}, p\right)$ to check. For instance, we have the prime candidate 2689 in the field of degree 96 corresponding to the character $\chi=\chi_{13}^{1} \chi_{97}^{9}$ (in an obvious notation). Since $2689 \equiv 1(\bmod 96)$, there are 96 minimal submodules corresponding to the various $\alpha_{i}=\eta^{\Phi_{96}(\sigma) /(\sigma-i)}$. We choose a pair $(q, b)$ and check the congruence (5.1). For instance, the pair $(74598239,46979)$ is appropriate. For this pair, the congruence (5.1) is not satisfied for any $\alpha_{i}$, thus $2689 \nmid h_{\chi}$. All the primes are checked similarly; 
we can handle all the primes not dividing the class number in this way. An example of a prime dividing the class number is given in the following.

Let $p=97$ and $\chi=\chi_{13}^{2} \chi_{97}^{10}$. We compute 10 appropriate pairs $(q, b)$ and notice that (5.1) is always satisfied for the minimal submodule corresponding to $f_{i}(\sigma)=$ $\sigma+48$ (the specific minimal submodule depends on the choice of the generator $\sigma$ of $G_{\chi}$; we had $\sigma$ defined by $\left.\zeta_{f} \mapsto \zeta_{f}^{19}\right)$. We move on to the third step and compute a real approximation of $\eta^{\Phi_{96}(\sigma) /(\sigma+48)}$ and its conjugates. Its minimum polynomial has huge coefficients, thus it is first important to reduce the coefficients of $\Phi_{96}(\sigma) /(\sigma+48) \in \mathbf{F}_{97}\left[G_{\chi}\right]$. Choosing the coefficients with the smallest absolute value modulo $p$ seems to be adequate; denote by $\alpha$ the element thus obtained. The precision we needed in this case was over 5000 digits in order to be able to compute the minimum polynomial $m(x)$ of $\alpha$. The choice of the coefficients of $\alpha$ was probably not ideal. Nevertheless, this was still possible to handle with a computer. The minimum polynomial $m_{p}(x)$ of $\sqrt[p]{\alpha}$ was computed in the same manner; it had much smaller coefficients, the largest with 54 digits. Finally, we checked that $m_{p}(x)$ divides $m\left(x^{p}\right)$. Moreover, we used the method of higher powers of $p$ to verify that $p^{2} \nmid h_{\chi}$.

There were altogether three pairs $\left(h_{\chi}, p\right)$ with $p$ not dividing $f$ (indeed, with $p=5$ or 7 ; see the table) for which we could not find any pairs $(q, b)$ failing to satisfy (5.1). They were all verified to be actual class number divisors using the third step.

The computing time of all the above was approximately one hour using Mathematica 4.1 24] on an AMD Athlon 2000+.

\section{Cohen-Lenstra heuristics}

Schoof [20] showed, based on a speculative extension of the Cohen-Lenstra heuristics [3], that the class numbers of real abelian fields of prime conductor are most likely relatively small. The same holds for prime power conductors; see Buhler et al. 2]. We see from Section 2 how to treat class groups of fields of any conductor. It would be natural to assume that the predictions given by Schoof on the size of the class groups hold in our case as well. We will show that this is indeed the case.

Cohen and Lenstra give conjectural heuristic assumptions on the properties of finite modules over direct products of Dedekind domains. In particular, the assumptions apply to the modules over the (unique) maximal order of the group ring $\mathbf{Q}[G] /\left\langle\sum_{\sigma \in G} \sigma\right\rangle$ with $G$ abelian. Their examples include probabilities for properties of the class groups of quadratic fields and real abelian fields. The $p$-parts of the class groups with $p$ dividing the degree had to be excluded; recently Wittmann 23 presented heuristics for such primes in some special cases.

To apply the heuristics, one should originally have a large collection of fields of varying conductor and fixed degree. Since our computations are limited to the fields of conductor at most 2000 and of varying degree, the situation is different. But as is mentioned in [2] and [20, the heuristics and the computed results together support the conjecture that the class groups of real abelian fields are usually very small.

We assume for the rest of the section that $p \nmid \# G$. The decomposition (2.2) allows us to define the $p$-class groups as modules over $\bigoplus_{\tilde{\chi} \neq \tilde{1}} \mathbf{Z}\left[\zeta_{g_{\chi}}\right]$; since $\mathrm{Cl}_{1, p}=1$ for the trivial character $1=\chi_{0}$, we may drop the corresponding part from the 
direct sum. Since the above sum is isomorphic to the maximal order of the group ring $\mathbf{Q}[G] /\left\langle\sum_{\sigma \in G} \sigma\right\rangle=\mathbf{Q}[G] / e_{1} \mathbf{Q}[G]$, the heuristics may be applied in our case.

For a finite module $A$ over a Dedekind domain $R$, there is a decomposition $A=\bigoplus_{\mathcal{P}} A_{\mathcal{P}}$, where the sum is taken over the prime ideals $\mathcal{P}$ of $R$ and

$$
A_{\mathcal{P}}=\left\{a \in A \mid \operatorname{Ann}_{R} a \text { is a power of } \mathcal{P}\right\} .
$$

Only finitely many $A_{\mathcal{P}} \neq 0$. Now by [3, Example 5.10], assuming the heuristics, the probability that $A_{\mathcal{P}}=0$ is equal to $\prod_{k=2}^{\infty}\left(1-N \mathcal{P}^{-k}\right)$, where the norm $N \mathcal{P}=$ $\#(A / \mathcal{P})$. The probabilities for the different $\mathcal{P}$ will be assumed independent.

Let us show how to apply the above probability in our case. Note first that the prime ideals of $\bigoplus_{\tilde{\chi} \neq \tilde{1}} \mathbf{Z}\left[\zeta_{g_{\chi}}\right]$ are of the form $\bigoplus_{\tilde{\chi} \neq \tilde{1}, \tilde{\psi}} \mathbf{Z}\left[\zeta_{g_{\chi}}\right] \oplus \mathcal{P}$, where $\widetilde{\psi}$ is any nontrivial $\mathbf{Q}$-conjugacy class of characters and $\mathcal{P}$ runs through the prime ideals of $\mathbf{Z}\left[\zeta_{g_{\psi}}\right]$. Their norms are equal to the norms of $\mathcal{P}$. There are $\varphi\left(g_{\chi}\right) / f_{p}$ prime ideals of $\mathbf{Z}\left[\zeta_{g_{\chi}}\right]$ above any unramified prime $p$ and their common norm is $p^{f_{p}}$, where $f_{p}$ is the order of $p$ modulo $g_{\chi}$. The number of different $\mathbf{Z}\left[\zeta_{g_{\chi}}\right]$ in the decomposition of the rational group ring of a real cyclotomic field is equal to the number of $\mathbf{Q}$ conjugacy classes. Their number might be calculated, for instance, by the following result by Perlis and Walker [19: If $G$ is a finite abelian group of order $g$, we have $\mathbf{Q}[G] \simeq \bigoplus_{d \mid g} \frac{n_{d}}{\varphi(d)} \mathbf{Q}\left(\zeta_{d}\right)$, where $n_{d}$ is the number of elements of order $d$ in $G$.

The probability that the class group is trivial (excluding the primes dividing $\left.2 g_{\chi}\right)$ is therefore

$$
P(\mathrm{Cl}=1)=\prod_{\tilde{\chi}} \prod_{p \in \mathbf{P}^{\prime}} \prod_{\mathcal{P} \mid p} P\left(\mathrm{Cl}_{\chi, \mathcal{P}}=1\right)=\prod_{\tilde{\chi}} \prod_{p \in \mathbf{P}^{\prime}}\left(\prod_{k \geq 2}\left(1-p^{-k f_{p}}\right)\right)^{\varphi\left(g_{\chi}\right) / f_{p}},
$$

where $\mathbf{P}^{\prime}$ denotes the set of all prime numbers $p \nmid 2 g_{\chi}$. Having computed all the $p$-parts of the class groups for $2<p<10000$, we assume $p>10000$. Then by taking the logarithm and using the estimates

$$
-\ln \left(1-\frac{1}{p^{k f_{p}}}\right)<\frac{1+10^{-8}}{p^{k f_{p}}} \quad(k \geq 2), \quad \sum_{k \geq 2} p^{-k f_{p}}=\frac{1}{p^{f_{p}}\left(p^{f_{p}}-1\right)} \leq \frac{1+10^{-4}}{p^{2 f_{p}}}
$$

we obtain

$$
-\ln \left(P\left(\mathrm{Cl}_{\chi, p}=1 \quad \forall p>10^{4}\right)\right)<1.00011 \varphi\left(g_{\chi}\right) \sum_{p>10^{4}} \frac{1}{f_{p} p^{2 f_{p}}} .
$$

The series is dominated by the terms with $f_{p}=1$, i.e., $p \equiv 1\left(\bmod g_{\chi}\right)$; the remainder is smaller than $\sum_{p>10^{4}} p^{-4}<10^{-13}$ (this is estimated via the "prime zeta function" (9.1)). By the prime number theorem for arithmetic progressions, the number of primes $p<n$ satisfying $p \equiv 1\left(\bmod g_{\chi}\right)$ equals approximately $\#\{p \in \mathbf{P} \mid p<n\} / \varphi\left(g_{\chi}\right)$ for large $n$. Thus with many different $g_{\chi}$ we have, at least on average,

$$
\sum_{p>10^{4}} \frac{1}{f_{p} p^{2 f_{p}}}<10^{-13}+\sum_{\substack{p>10^{4} \\ p \equiv 1}} p^{-2} \approx \frac{1}{\varphi\left(g_{\chi}\right)} \sum_{p>10^{4}} p^{-2} .
$$


We assumed that $10^{-13}$ is insignificant; this holds, when the numbers $g_{\chi}$ are of the magnitude we confronted in the computations. The series over primes may be approximated from its expression in terms of values $\zeta(m)$ of the Riemann zeta function, $m \geq 2$. Indeed, we have

$$
\sum_{p \in \mathbf{P}} \frac{1}{p^{m}}=\sum_{k=1}^{\infty} \frac{\mu(k)}{k} \ln \zeta(k m)
$$

as the Möbius inversion of the logarithm of the Euler product for $\zeta(m)$ (see, e.g., [4]). This gives $\sum_{p \in \mathbf{P}} p^{-2} \approx 0.452247$. Consequently, we obtain $\sum_{p<10^{4}} p^{-2} \approx 0.452238$. It follows that

$$
P\left(\mathrm{Cl}_{\chi, p}=1 \quad \forall p>10^{4}\right) \approx 0.999990 .
$$

It is interesting to note that this estimate does not depend on $g_{\chi}$.

We computed all the $(\chi, p)$-parts of the class groups for $2<p<10000, p \nmid g_{\chi}$, $f_{\chi} \leq 2000$. For $f_{\chi} \leq 500$, we even went up to the bound $p<100000$ utilizing Schwarz's tables [21]. For any fixed $p$, there are a total of 9339 different $\mathbf{Z}\left[\zeta_{g_{\chi}}\right]$ modules $\mathrm{Cl}_{\chi, p}$ for $500<f \leq 2000$ (1679 for $f_{\chi} \leq 500$ ). When substituting this information in the above formulas, one obtains from the heuristics that the predicted number of occurrences of nontrivial class group parts $\mathrm{Cl}_{\chi, p}$ (dropping out from the study all the primes dividing $2 g_{\chi}$ ) for the fields of conductor $f_{\chi} \leq 2000$ would be approximately 443 , and that the class number would not contain larger primes for $500<f_{\chi} \leq 2000$ with probability $\approx 91 \%$ (for $f_{\chi} \leq 500$ with $\approx 99 \%$ ). We might exclude from the calculation all the class group parts corresponding to the fields of small degree since there exist extensive tables for them; then the above probability for $500<f_{\chi} \leq 2000$ rises to at least $93 \%$. Given that all the computations have produced only relatively small prime divisors compared to the degree of the field, we find it reasonable to believe that the class number divisors found are, in fact, all the primes dividing $h_{\chi}$ for any $f_{\chi} \leq 2000$, excluding the primes dividing $2 g_{\chi}$.

We found 231 nontrivial $\chi$-parts of class groups, which is less than the expected number 443 , but which is still of the same order of magnitude when compared to the number of all the $\chi$-parts. This supports the belief, stated by Schoof 20, that the heuristics would slightly overestimate the chance of a nontrivial class group when the conductor is relatively small.

\section{TABLE}

In the enclosed table we present all the prime divisors $2<p<10000$ of the class numbers of the real abelian fields of composite conductor $500<f \leq 2000$ and the prime divisors $p<100000$ for $f \leq 500$, excluding the primes dividing the degree of the field. The first column indicates the conductor $f_{\chi}$ of $K_{\chi}$. A character defining the field $K_{\chi}$ is written in the second column. We use the notation $\chi_{\ell^{\nu}}$ for the generating character modulo $\ell^{\nu}$ with $\ell>2$ a prime. Let $\omega_{4}$ modulo 4 be defined by $\omega_{4}(-1)=-1$. For $\nu \geq 3$, define $\chi_{2^{\nu}}$ modulo $2^{\nu}$ by $\chi_{2^{\nu}}(5)=\zeta_{2^{\nu-2}}$ and $\chi_{2^{\nu}}(-1)=1$. The representatives of the $\mathbf{Q}$-conjugacy classes of characters were chosen as in [21].

The third column gives the degree $g_{\chi}$ of $K_{\chi}$ and the last column shows the prime divisor $p$ of the $\chi$-class number $h_{\chi}$. We did not encounter any $h_{\chi}$ having more than one prime divisor. The occasional exponent of $p$ is the residue class degree of $p$ modulo $g_{\chi}$, except for one case. This is a field of conductor 1921 for 
which we found two different submodules containing 17th powers. The search for higher powers of $p$ showed that the class number is exactly divisible by $17^{3}$. We computed, using PARI [18, that the 17-class group is of type $\mathbf{Z} / 17^{2} \mathbf{Z} \times \mathbf{Z} / 17 \mathbf{Z}$. Note that 17 divides 1921. In general, the case where $p$ divides the conductor seems to occur very often. For the fields of prime power conductor, recall that Vandiver's conjecture (verified up to a very large conductor) states that such primes never divide the class numbers.

For any real field $K$ of conductor $f$, one may read the $p$-part of $h_{K}$ for any $p<10000, p \nmid 2[K: \mathbf{Q}]$, by combining the entries of the table (together with Schoof's table of the fields of prime conductor in [20]) for all the cyclic subfields $K_{\chi}$ of $K$ of conductor $f_{\chi} \mid f$. The $p$-class structure is given by (2.2).

For example, let $K=\mathbf{Q}\left(\zeta_{f}+\zeta_{f}^{-1}\right)$ with $f=1304=8 \cdot 163$. Our table gives for $h_{K}$ twice the prime factor 19 coming from fields with conductor $f$ and $f / 2=652$ (both of degree 18). By (2.2), the 19-class group is of type $\mathbf{Z} / 19 \mathbf{Z} \times \mathbf{Z} / 19 \mathbf{Z}$. In addition, there is a prime factor 3 coming from a quadratic subfield with conductor $f$. Since 3 divides the degree 324 of $K$, the 3 -class group of $K$ remains unknown; in fact, it could be possible that $3 \nmid h_{K}$ 1 . Since the class number of $\mathbf{Q}\left(\zeta_{8}+\zeta_{8}^{-1}\right)$ is 1 and that of $\mathbf{Q}\left(\zeta_{163}+\zeta_{163}^{-1}\right)$ is 4 (see [14]), we find that all the other possible odd prime factors of $h_{K}$ must be larger than 10000 .

The results were checked to agree with the tables of real cyclic fields of degree at most 6 (cf. [17, [6], 7], 9], [15). All the class number divisors of the fields of degree at most 20 were also confirmed with PARI. The results in the case of a prime conductor (omitted from this table) were found to agree with the tables of Schoof [20] and Koyama and Yoshino [11].

\footnotetext{
${ }^{1}$ One can show that, in fact, $3 \mid h_{K}$ (personal communication by C. Greither).
} 
TUOMAS HAKKARAINEN

\begin{tabular}{|c|c|c|c|c|c|c|c|c|c|c|c|}
\hline$f_{\chi}$ & $\chi$ & $g_{\chi}$ & $p$ & $f_{\chi}$ & $\chi$ & $g_{\chi}$ & $p$ & $f_{\chi}$ & $\chi$ & $g_{\chi}$ & $p$ \\
\hline 212 & $\omega_{4}^{1} \chi_{53}^{13}$ & 4 & 5 & 976 & $\omega_{4}^{1} \chi_{16}^{1} \chi_{61}^{15}$ & 4 & 5 & 1311 & $\chi_{3}^{1} \chi_{19}^{2} \chi_{23}^{11}$ & 18 & 19 \\
\hline 316 & $\omega_{4}^{1} \chi_{79}^{39}$ & 2 & 3 & 980 & $\omega_{4}^{1} \chi_{5}^{1} \chi_{49}^{6}$ & 28 & 29 & 1313 & $\chi_{13}^{6} \chi_{101}^{20}$ & 10 & 31 \\
\hline 321 & $\chi_{3}^{1} \chi_{107}^{53}$ & 2 & 3 & 985 & $\chi_{5}^{2} \chi_{197}^{98}$ & 2 & 3 & 1332 & $\omega_{4}^{1} \chi_{9}^{1} \chi_{37}^{6}$ & 6 & 7 \\
\hline 427 & $\chi_{7}^{3} \chi_{61}^{15}$ & 4 & 5 & 988 & $\omega_{4}^{1} \chi_{13}^{2} \chi_{19}^{3}$ & 6 & 7 & 1339 & $\chi_{13}^{3} \chi_{103}^{17}$ & 12 & 13 \\
\hline 469 & $\chi_{7}^{3} \chi_{67}^{33}$ & 2 & 3 & 993 & $\chi_{3}^{1} \chi_{331}^{165}$ & 2 & 3 & 1343 & $\chi_{17}^{1} \chi_{79}^{39}$ & 16 & 17 \\
\hline 473 & $\chi_{11}^{5} \chi_{43}^{21}$ & 2 & 3 & 999 & $\chi_{27}^{2} \chi_{37}^{16}$ & 9 & 37 & 1345 & $\chi_{5}^{2} \chi_{269}^{134}$ & 2 & 3 \\
\hline 481 & $\chi_{13}^{2} \chi_{37}^{4}$ & 18 & 19 & 1016 & $\omega_{4}^{1} \chi_{8}^{1} \chi_{127}^{63}$ & 2 & 3 & 1353 & $\chi_{3}^{1} \chi_{11}^{1} \chi_{41}^{12}$ & 10 & 11 \\
\hline 551 & $\chi_{19}^{9} \chi_{29}^{7}$ & 4 & 5 & 1025 & $\chi_{25}^{1} \chi_{41}^{7}$ & 40 & 41 & 1355 & $\chi_{5}^{2} \chi_{271}^{30}$ & 18 & 37 \\
\hline 556 & $\omega_{4}^{1} \chi_{139}^{23}$ & 6 & 7 & 1036 & $\omega_{4}^{1} \chi_{7}^{2} \chi_{37}^{5}$ & 36 & 73 & 1359 & $\chi_{9}^{1} \chi_{151}^{125}$ & 6 & 7 \\
\hline \multirow[t]{2}{*}{568} & $\chi_{8}^{1} \chi_{71}^{14}$ & 10 & 11 & 1048 & $\chi_{8}^{1} \chi_{131}^{26}$ & 10 & 11 & 1360 & $\omega_{4}^{1} \chi_{16}^{1} \chi_{5}^{1} \chi_{17}^{12}$ & 4 & 5 \\
\hline & $\omega_{4}^{1} \chi_{8}^{1} \chi_{71}^{35}$ & 2 & 3 & 1080 & $\chi_{8}^{1} \chi_{27}^{1} \chi_{5}^{1}$ & 36 & 37 & 1376 & $\omega_{4}^{1} \chi_{32}^{1} \chi_{43}^{7}$ & 24 & $5^{2}$ \\
\hline \multirow[t]{2}{*}{629} & $\chi_{17}^{8} \chi_{37}^{2}$ & 18 & 19 & 1101 & $\chi_{3}^{1} \chi_{367}^{183}$ & 2 & 3 & 1384 & $\chi_{8}^{1} \chi_{173}^{86}$ & 2 & 3 \\
\hline & $\chi_{17}^{4} \chi_{37}^{18}$ & 4 & 5 & 1105 & $\chi_{5}^{1} \chi_{13}^{9} \chi_{17}^{8}$ & 4 & 5 & 1385 & $\chi_{5}^{2} \chi_{277}^{46}$ & 6 & 7 \\
\hline 651 & $\chi_{3}^{1} \chi_{7}^{3} \chi_{31}^{6}$ & 10 & 11 & 1113 & $\chi_{3}^{1} \chi_{7}^{2} \chi_{53}^{13}$ & 12 & 13 & & $\chi_{5}^{1} \chi_{277}^{207}$ & 4 & 5 \\
\hline 652 & $\omega_{4}^{1} \chi_{163}^{9}$ & 18 & 19 & 1116 & $\omega_{4}^{1} \chi_{9}^{2} \chi_{31}^{25}$ & 6 & 7 & 1387 & $\chi_{19}^{2} \chi_{73}^{18}$ & 36 & $17^{2}$ \\
\hline 676 & $\omega_{4}^{1} \chi_{169}^{3}$ & 52 & 53 & 1132 & $\omega_{4}^{1} \chi_{283}^{47}$ & 6 & 7 & & $\chi_{19}^{2} \chi_{73}^{22}$ & 36 & 37 \\
\hline 692 & $\omega_{4}^{1} \chi_{173}^{43}$ & 4 & 5 & 1139 & $\chi_{17}^{2} \chi_{67}^{6}$ & 88 & 89 & & $\chi_{19}^{2} \chi_{73}^{8}$ & 9 & 19 \\
\hline 697 & $\chi_{17}^{8} \chi_{41}^{20}$ & 2 & 3 & 1141 & $\chi_{7}^{2} \chi_{163}^{36}$ & 9 & 19 & 1393 & $\chi_{7}^{3} \chi_{199}^{99}$ & 2 & 5 \\
\hline \multirow[t]{2}{*}{703} & $\chi_{19}^{9} \chi_{37}^{1}$ & 36 & 37 & 1159 & $\chi_{19}^{2} \chi_{61}^{10}$ & 18 & 73 & 1404 & $\omega_{4}^{1} \chi_{27}^{1} \chi_{13}^{8}$ & 18 & 19 \\
\hline & $\chi_{19}^{3} \chi_{37}^{9}$ & 12 & 13 & 1172 & $\omega_{4}^{1} \chi_{293}^{73}$ & 4 & 13 & 1407 & $\chi_{3}^{1} \chi_{7}^{3} \chi_{67}^{6}$ & 22 & 23 \\
\hline 728 & $\chi_{8}^{1} \chi_{7}^{3} \chi_{13}^{3}$ & 4 & 5 & 1197 & $\chi_{9}^{2} \chi_{7}^{5} \chi_{19}^{15}$ & 6 & 7 & 1420 & $\omega_{4}^{1} \chi_{5}^{2} \chi_{71}^{7}$ & 10 & 11 \\
\hline 753 & $\chi_{3}^{1} \chi_{251}^{25}$ & 10 & 11 & 1207 & $\chi_{17}^{1} \chi_{71}^{35}$ & 16 & 17 & 1421 & $\chi_{49}^{3} \chi_{29}^{11}$ & 28 & 29 \\
\hline 756 & $\omega_{4}^{1} \chi_{27}^{2} \chi_{7}^{1}$ & 18 & 19 & 1211 & $\chi_{7}^{2} \chi_{173}^{86}$ & 6 & 7 & 1424 & $\omega_{4}^{1} \chi_{16}^{1} \chi_{89}^{11}$ & 8 & 17 \\
\hline 763 & $\chi_{7}^{3} \chi_{109}^{9}$ & 12 & 13 & 1235 & $\chi_{5}^{1} \chi_{13}^{4} \chi_{19}^{15}$ & 12 & 13 & 1435 & $\chi_{5}^{1} \chi_{7}^{1} \chi_{41}^{10}$ & 12 & 13 \\
\hline 779 & $\chi_{19}^{9} \chi_{41}^{1}$ & 40 & 41 & & $\chi_{5}^{2} \chi_{13}^{3} \chi_{19}^{9}$ & 4 & 5 & 1436 & $\omega_{4}^{1} \chi_{359}^{179}$ & 2 & 3 \\
\hline 785 & $\chi_{5}^{2} \chi_{157}^{78}$ & 2 & 3 & 1241 & $\chi_{17}^{4} \chi_{73}^{18}$ & 4 & 5 & 1455 & $\chi_{3}^{1} \chi_{5}^{1} \chi_{97}^{6}$ & 16 & 17 \\
\hline 793 & $\chi_{13}^{1} \chi_{61}^{55}$ & 12 & 37 & 1243 & $\chi_{11}^{2} \chi_{113}^{14}$ & 40 & 41 & 1460 & $\omega_{4}^{1} \chi_{5}^{1} \chi_{73}^{54}$ & 4 & 5 \\
\hline 808 & $\omega_{4}^{1} \chi_{8}^{1} \chi_{101}^{25}$ & 4 & 5 & 1257 & $\chi_{3}^{1} \chi_{419}^{209}$ & 2 & 3 & 1461 & $\chi_{3}^{1} \chi_{487}^{27}$ & 18 & 19 \\
\hline 817 & $\chi_{19}^{9} \chi_{43}^{21}$ & 2 & 5 & 1261 & $\chi_{13}^{2} \chi_{97}^{10}$ & 48 & 97 & 1465 & $\chi_{5}^{1} \chi_{293}^{219}$ & 4 & $3^{2}$ \\
\hline 819 & $\chi_{9}^{1} \chi_{7}^{1} \chi_{13}^{2}$ & 6 & 7 & & $\chi_{13}^{2} \chi_{97}^{64}$ & 6 & 7 & 1477 & $\chi_{7}^{3} \chi_{211}^{21}$ & 10 & 11 \\
\hline 832 & $\omega_{4}^{1} \chi_{64}^{1} \chi_{13}^{3}$ & 16 & $7^{2}$ & & $\chi_{13}^{6} \chi_{97}^{24}$ & 4 & 5 & & $\chi_{7}^{1} \chi_{211}^{35}$ & 6 & 7 \\
\hline 869 & $\chi_{11}^{5} \chi_{79}^{1}$ & 78 & 79 & & $\chi_{13}^{4} \chi_{97}^{64}$ & 3 & 7 & 1496 & $\omega_{4}^{1} \chi_{8}^{1} \chi_{11}^{1} \chi_{17}^{8}$ & 10 & 11 \\
\hline 889 & $\chi_{7}^{3} \chi_{127}^{21}$ & 6 & 7 & 1271 & $\chi_{31}^{2} \chi_{41}^{24}$ & 15 & 31 & 1509 & $\chi_{3}^{1} \chi_{503}^{251}$ & 2 & 3 \\
\hline 892 & $\omega_{4}^{1} \chi_{223}^{111}$ & 2 & 3 & & $\chi_{31}^{10} \chi_{41}^{20}$ & 6 & 7 & 1513 & $\chi_{17}^{1} \chi_{89}^{11}$ & 16 & 17 \\
\hline 916 & $\omega_{4}^{1} \chi_{229}^{57}$ & 4 & 5 & & $\chi_{31}^{6} \chi_{41}^{24}$ & 5 & 11 & & $\chi_{17}^{8} \chi_{89}^{22}$ & 4 & 13 \\
\hline 923 & $\chi_{13}^{3} \chi_{71}^{7}$ & 20 & 61 & 1287 & $\chi_{9}^{1} \chi_{11}^{2} \chi_{13}^{3}$ & 60 & 61 & 1516 & $\omega_{4}^{1} \chi_{379}^{1}$ & 378 & 379 \\
\hline 928 & $\omega_{4}^{1} \chi_{32}^{1} \chi_{29}^{7}$ & 8 & 17 & 1295 & $\chi_{5}^{2} \chi_{7}^{2} \chi_{37}^{10}$ & 18 & 19 & 1525 & $\chi_{25}^{2} \chi_{61}^{24}$ & 10 & 11 \\
\hline 935 & $\chi_{5}^{1} \chi_{11}^{5} \chi_{17}^{4}$ & 4 & 5 & 1304 & $\chi_{8}^{1} \chi_{163}^{18}$ & 18 & 19 & 1547 & $\chi_{7}^{1} \chi_{13}^{1} \chi_{17}^{12}$ & 12 & 37 \\
\hline 940 & $\omega_{4}^{1} \chi_{5}^{2} \chi_{47}^{23}$ & 2 & 3 & & $\omega_{4}^{1} \chi_{8}^{1} \chi_{163}^{81}$ & 2 & 3 & 1575 & $\chi_{9}^{1} \chi_{25}^{2} \chi_{7}^{5}$ & 30 & 31 \\
\hline 944 & $\omega_{4}^{1} \chi_{16}^{1} \chi_{59}^{29}$ & 4 & 5 & 1308 & $\omega_{4}^{1} \chi_{3}^{1} \chi_{109}^{18}$ & 6 & 7 & 1576 & $\omega_{4}^{1} \chi_{8}^{1} \chi_{197}^{49}$ & 4 & $3^{2}$ \\
\hline
\end{tabular}




\begin{tabular}{|c|c|c|c|c|c|c|c|}
\hline$f_{\chi}$ & $\chi$ & $g_{\chi}$ & $p$ & $f_{\chi}$ & $\chi$ & $g_{\chi}$ & $p$ \\
\hline 1591 & $\chi_{37}^{18} \chi_{43}^{2}$ & 42 & 43 & 1855 & $\chi_{5}^{2} \chi_{7}^{3} \chi_{53}^{13}$ & 4 & 5 \\
\hline \multirow[t]{2}{*}{1592} & $\omega_{4}^{1} \chi_{8}^{1} \chi_{199}^{11}$ & 18 & 19 & 1865 & $\chi_{5}^{1} \chi_{373}^{93}$ & 4 & 5 \\
\hline & $\omega_{4}^{1} \chi_{8}^{1} \chi_{199}^{33}$ & 6 & 7 & 1872 & $\chi_{16}^{1} \chi_{9}^{2} \chi_{13}^{10}$ & 12 & 13 \\
\hline 1620 & $\omega_{4}^{1} \chi_{81}^{2} \chi_{5}^{1}$ & 108 & 109 & 1885 & $\chi_{5}^{1} \chi_{13}^{6} \chi_{29}^{1}$ & 28 & 29 \\
\hline 1623 & $\chi_{3}^{1} \chi_{541}^{45}$ & 12 & 13 & & $\chi_{5}^{2} \chi_{13}^{3} \chi_{29}^{3}$ & 28 & 113 \\
\hline \multirow[t]{2}{*}{1629} & $\chi_{9}^{2} \chi_{181}^{18}$ & 30 & 31 & & $\chi_{5}^{1} \chi_{13}^{6} \chi_{29}^{7}$ & 4 & 5 \\
\hline & $\chi_{9}^{2} \chi_{181}^{50}$ & 18 & 109 & 1887 & $\chi_{3}^{1} \chi_{17}^{4} \chi_{37}^{27}$ & 4 & 5 \\
\hline 1640 & $\omega_{4}^{1} \chi_{8}^{1} \chi_{5}^{2} \chi_{41}^{5}$ & 8 & $3^{2}$ & 1891 & $\chi_{31}^{3} \chi_{61}^{21}$ & 20 & 41 \\
\hline 1641 & $\chi_{3}^{1} \chi_{547}^{273}$ & 2 & 5 & & $\chi_{31}^{2} \chi_{61}^{28}$ & 15 & 31 \\
\hline 1643 & $\chi_{31}^{5} \chi_{53}^{13}$ & 12 & 13 & & $\chi_{31}^{6} \chi_{61}^{6}$ & 10 & 11 \\
\hline 1651 & $\chi_{13}^{1} \chi_{127}^{63}$ & 12 & $5^{2}$ & 1897 & $\chi_{7}^{3} \chi_{271}^{135}$ & 2 & 5 \\
\hline 1665 & $\chi_{9}^{1} \chi_{5}^{1} \chi_{37}^{24}$ & 12 & 13 & 1903 & $\chi_{11}^{5} \chi_{173}^{1}$ & 172 & 173 \\
\hline 1676 & $\omega_{4}^{1} \chi_{419}^{19}$ & 22 & 23 & 1904 & $\chi_{16}^{1} \chi_{7}^{3} \chi_{17}^{3}$ & 16 & 97 \\
\hline 1687 & $\chi_{7}^{2} \chi_{241}^{80}$ & 3 & 13 & & $\omega_{4}^{1} \chi_{16}^{1} \chi_{7}^{1} \chi_{17}^{12}$ & 12 & 13 \\
\hline 1688 & $\chi_{8}^{1} \chi_{211}^{42}$ & 10 & 31 & 1921 & $\chi_{17}^{4} \chi_{113}^{8}$ & 28 & 29 \\
\hline \multirow[t]{2}{*}{1708} & $\omega_{4}^{1} \chi_{7}^{1} \chi_{61}^{50}$ & 6 & 7 & & $\chi_{17}^{1} \chi_{113}^{35}$ & 16 & $17 \cdot 17^{2}$ \\
\hline & $\omega_{4}^{1} \chi_{7}^{3} \chi_{61}^{30}$ & 2 & 3 & 1929 & $\chi_{3}^{1} \chi_{643}^{321}$ & 2 & 3 \\
\hline \multirow[t]{3}{*}{1729} & $\chi_{7}^{2} \chi_{13}^{3} \chi_{19}^{3}$ & 12 & $5^{2}$ & 1935 & $\chi_{9}^{2} \chi_{5}^{1} \chi_{43}^{7}$ & 12 & 13 \\
\hline & $\chi_{7}^{1} \chi_{13}^{5} \chi_{19}^{12}$ & 12 & 13 & & $\chi_{9}^{2} \chi_{5}^{1} \chi_{43}^{21}$ & 12 & 13 \\
\hline & $\chi_{7}^{1} \chi_{13}^{2} \chi_{19}^{15}$ & 6 & 7 & 1937 & $\chi_{13}^{1} \chi_{149}^{111}$ & 12 & 109 \\
\hline 1735 & $\chi_{5}^{1} \chi_{347}^{173}$ & 4 & 5 & & $\chi_{13}^{6} \chi_{149}^{74}$ & 2 & 3 \\
\hline 1736 & $\omega_{4}^{1} \chi_{8}^{1} \chi_{7}^{2} \chi_{31}^{15}$ & 6 & 7 & 1957 & $\chi_{19}^{9} \chi_{103}^{51}$ & 2 & 3 \\
\hline 1739 & $\chi_{37}^{9} \chi_{47}^{23}$ & 4 & 5 & 1965 & $\chi_{3}^{1} \chi_{5}^{2} \chi_{131}^{13}$ & 10 & 11 \\
\hline 1749 & $\chi_{3}^{1} \chi_{11}^{5} \chi_{53}^{2}$ & 26 & 53 & 1971 & $\chi_{27}^{2} \chi_{73}^{4}$ & 18 & 19 \\
\hline 1751 & $\chi_{17}^{1} \chi_{103}^{51}$ & 16 & 17 & 1972 & $\omega_{4}^{1} \chi_{17}^{2} \chi_{29}^{7}$ & 8 & $3^{2}$ \\
\hline 1755 & $\chi_{27}^{2} \chi_{5}^{1} \chi_{13}^{3}$ & 36 & 73 & 1976 & $\chi_{8}^{1} \chi_{13}^{6} \chi_{19}^{2}$ & 18 & 19 \\
\hline 1756 & $\omega_{4}^{1} \chi_{439}^{219}$ & 2 & 5 & & $\chi_{8}^{1} \chi_{13}^{1} \chi_{19}^{3}$ & 12 & 13 \\
\hline 1761 & $\chi_{3}^{1} \chi_{587}^{293}$ & 2 & 7 & 1988 & $\omega_{4}^{1} \chi_{7}^{2} \chi_{71}^{5}$ & 42 & 43 \\
\hline 1765 & $\chi_{5}^{2} \chi_{353}^{176}$ & 2 & 3 & & $\omega_{4}^{1} \chi_{7}^{1} \chi_{71}^{14}$ & 30 & 31 \\
\hline 1772 & $\omega_{4}^{1} \chi_{443}^{221}$ & 2 & 3 & 1995 & $\chi_{3}^{1} \chi_{5}^{2} \chi_{7}^{2} \chi_{19}^{3}$ & 6 & 7 \\
\hline 1853 & $\chi_{17}^{8} \chi_{109}^{6}$ & 18 & 19 & 1996 & $\omega_{4}^{1} \chi_{499}^{249}$ & 2 & 5 \\
\hline
\end{tabular}




\section{ACKNOWLEDGMENT}

The author wishes to thank Professor Tauno Metsänkylä for his advice and support.

\section{REFERENCES}

1. M. Aoki, Notes on the structure of the ideal class groups of abelian number fields, Proc. Japan Acad. Ser. A Math. Sci. 81 (2005), no. 5, pp. 69-74. MR2143545(2006a:11142)

2. J. Buhler, C. Pomerance, L. Robertson, Heuristics for class numbers of prime-power real cyclotomic fields, High primes and misdemeanours: lectures in honour of the 60th birthday of Hugh Cowie Williams, Fields Inst. Commun. 41, Amer. Math. Soc., Providence, RI (2004), pp. 149-157. MR2073643 (2005e:11143)

3. H. Cohen, H. W. Lenstra, Heuristics on class groups of number fields, Number theory, Noordwijkerhout 1983 (Noordwijkerhout, 1983), Lecture Notes in Math. 1068, Springer, Berlin (1984), pp. 33-62. MR756082 (85j:11144)

4. C.-E. Fröberg, On the prime zeta function, BIT 8 (1968), pp. 187-202. MR0236123 (38:4421)

5. G. Gras and M.-N. Gras, Calcul du nombre de classes et des unités des extensions abéliennes réelles de Q, Bull. Sci. Math. (2) 101 (1977), no. 2, pp. 97-129. MR0480423 (58:586)

6. M.-N. Gras, Méthodes et algorithmes pour le calcul numérique du nombre de classes et des unités des extensions cubiques cycliques de Q, J. Reine Angew. Math. 277 (1975), pp. 89-116. MR0389845 (52:10675)

7. M.-N. Gras, Table numérique du nombre de classes et des unités dans les extensions cycliques réelles de degre 4 de Q, Publ. Math. Fac. Sci. Besançon 1977/78, Fasc. 2 (1978), 52 pp.

8. T. Hakkarainen, On the computation of the class numbers of real abelian fields, TUCS Dissertations no. 87, Turku Centre for Computer Science (2007), 81 pp. Available at http://www. tucs.fi/

9. S. Jeannin, Tables des nombres de classes et unités des corps quintiques cycliques de conducteur $f \leq 10000$, Publ. Math. Fac. Sci. Besançon 1994/95-1995/96 (1997), 40 pp. MR1449427 (98b:11129)

10. S. Kobayashi, Divisibilité du nombre de classes des corps abéliens réels, J. Reine Angew. Math. 320 (1980), pp. 142-149. MR592150 (82f:12009)

11. Y. Koyama and K. Yoshino, Prime divisors of real class number of $p^{r}$ th cyclotomic field and characteristic polynomials attached to them, Preprint (2003), $23 \mathrm{pp}$.

12. H. W. Leopoldt, Über Einheitengruppe und Klassenzahl reeller abelscher Zahlkörper, Abh. Deutsch. Akad. Wiss. Berlin. Kl. Math. Nat. 1953, no. 2 (1954), 48 pp. MR0067927 (16:799d)

13. H. W. Leopoldt, Über Klassenzahlprimteiler reeller abelscher Zahlkörper als Primteiler verallgemeinerter Bernoullischer Zahlen, Abh. Math. Sem. Univ. Hamburg 23 (1959), pp. 36-47. MR0103184 (21:1967)

14. F. van der Linden, Class number computations of real abelian number fields, Math. Comp. 39 (1982), pp. 693-707. MR669662 (84e:12005)

15. S. Mäki, The determination of units in real cyclic sextic fields, Lecture Notes in Math. 797, Springer, Berlin (1980), 198 pp. MR.584794 (82a:12004)

16. T. Metsänkylä, An application of the p-adic class number formula, Manuscripta Math. 93 (1997), pp. 481-498. MR1465893 (98m:11118)

17. B. Oriat, Groupes des classes d'idéaux des corps quadratiques réels $\mathbf{Q}\left(d^{1 / 2}\right), 1<d<$ 24572, Publ. Math. Fac. Sci. Besançon 1986/87-1987/88, Fasc. 2 (1988), 65 pp. MR983124 (90e:11167a)

18. PARI/GP, version 2.2.8, Bordeaux, 2005, http://pari.math.u-bordeaux.fr/

19. S. Perlis and G. Walker, Abelian group algebras of finite order, Trans. Amer. Math. Soc. 68 (1950), pp. 420-426. MR0034758 (11:638k)

20. R. Schoof, Class numbers of real cyclotomic fields of prime conductor, Math. Comp. 72 (2003), pp. 913-937. MR.1954975 (2004f:11116)

21. W. Schwarz, Über die Klassenzahl abelscher Zahlkörper, Ph.D. Thesis, University of Saarbrücken (1995), 125 pp.

22. L. Washington, Introduction to Cyclotomic Fields, 2nd ed., Springer, New York, 1997. MR:1421575 (97h:11130) 
23. C. Wittmann, p-class groups of certain extensions of degree p, Math. Comp. 74 (2005), pp. 937-947. MR2114656 (2005h:11256)

24. Wolfram Research, Inc., Mathematica, Version 4.1, Champaign, IL (2001).

Department of Mathematics \& TuCS, Turku Centre for Computer Science, UniverSity of Turku, FI-20014 Turku, Finland 